\title{
Size matters: A comparison of T1 and T2 peripheral non-small-cell lung cancers treated with stereotactic body radiation therapy (SBRT)
}

\author{
Neal E. Dunlap, MD, ${ }^{\mathrm{a}}$ James M. Larner, MD, ${ }^{\mathrm{a}}$ Paul W. Read, MD, PhD, ${ }^{\mathrm{a}}$ Benjamin D. Kozower, MD, \\ Christine L. Lau, MD, ${ }^{\mathrm{b}}$ Ke Sheng, PhD, ${ }^{\mathrm{a}}$ and David R. Jones, $\mathrm{MD}^{\mathrm{b}}$
}

Objective: The purpose of this study was to compare the outcomes and local control rates of patients with peripheral T1 and T2 non-small-cell lung cancer treated with stereotactic body radiation therapy.

\begin{abstract}
Methods: The records of 40 consecutive patients treated with 3- or 5-fraction lung stereotactic body radiation therapy for peripheral, clinical stage I non-small-cell lung cancer were reviewed. Stereotactic body radiation therapy was delivered at a median dose of $60 \mathrm{~Gy}$. Doses to organs at risk were limited based on the Radiation Therapy Oncology Group 0236 treatment protocol. Patients were staged clinically. Median follow was 12.5 months.
\end{abstract}

\begin{abstract}
Results: Twenty-seven (67\%) patients and $13(33 \%)$ patients had T1 and T2 tumors, respectively. Thirty-seven $(94 \%)$ patients were medically inoperable. Nine $(23 \%)$ patients had chest wall pain after stereotactic body radiation therapy. Symptomatic pneumonitis developed in $4(10 \%)$ patients. Increasing tumor size correlated with worse local control and overall survival. The median recurrence-free survival for T1 and T2 tumors was 30.6 months (95\% confidence interval [CI], 26.9-34.2) and 20.5 months (95\% CI, 14.3-26.5), respectively $(P=.038)$. Local control at 2 years was $90 \%$ and $70 \%$ in $\mathrm{T} 1$ and $\mathrm{T} 2$ tumors, respectively $(P=.03)$. The median survival for T1 and T2 tumors was 20 months $(95 \% \mathrm{CI}, 20.1-31.6)$ and 16.7 months (95\% CI, 10.8-21.2), respectively $(P=.073)$.
\end{abstract}

Conclusions: Stereotactic body radiation therapy for T2 non-small-cell lung cancer has a higher local recurrence rate and trended toward a worse survival than did T1 lesions. Tumor size is an important predictor of response to stereotactic body radiation therapy and should be considered in treatment planning. (J Thorac Cardiovasc Surg 2010;140:583-9)

\section{INTRODUCTION}

Early-stage (T1-2 N0) primary non-small-cell lung cancer (NSCLC) traditionally has been managed with surgical resection. Anatomic surgical resection, usually with a lobectomy, for these early-stage tumors has resulted in 3- to 5 -year survivals of $60 \%$ to $80 \% .^{1,2}$ Unfortunately, significant complications are associated with lobectomy in elderly patients or those with medical comorbidities such as poor pulmonary reserve or cardiovascular disease. ${ }^{3,4}$ More limited surgical procedures (wedge resection or segmentectomy) may be used but are associated with poorer outcomes than are definitive surgical procedures. ${ }^{5}$ Historically, external beam radiation therapy has been used as a primary treatment modality for patients with early-stage NSCLC who are not operative candidates. This standard 3-dimensional conformal therapy approach uses small daily fractions of radiation of 1.8 to 2 Gy for a total

\footnotetext{
From the Departments of Surgery ${ }^{\mathrm{b}}$ and Radiation Oncology, ${ }^{\mathrm{a}}$ University of Virginia, Charlottesville, Va.

Received for publication June 20, 2009; revisions received Sept 17, 2009; accepted for publication Jan 12, 2010; available ahead of print May 17, 2010.

Address for reprints: David R. Jones, MD, Professor of Surgery, Department of Surgery, University of Virginia, PO Box 800709, Charlottesville, VA 22908 (E-mail: djones@virginia.edu).

0022-5223/\$36.00

Copyright (C) 2010 by The American Association for Thoracic Surgery

doi:10.1016/j.jtcvs.2010.01.046
}

dose of 50 to 66 Gy. Suboptimal 5-year survivals of $10 \%$ to $30 \%$ (median survival, 12-16 months) and 5-year local control rates of $50 \%$ to $70 \%$ have been reported with this technique. ${ }^{6,7}$

The recognition that dose escalation is an important component for improved tumor control led to the development of stereotactic body radiation therapy (SBRT). SBRT uses elements of 3-dimensional conformal therapy combined with stereotactic tumor localization to deliver significantly higher daily doses of radiation while limiting radiation dose to the surrounding normal tissues. Results from North America and Europe have shown 3-year local control rates of 70\% to $90 \%$ with 2 -year survivals of approximately $50 \%{ }^{8-10}$ Intuitively, tumor size would be expected to be an independent predictor of local failure. Prior prospective studies using lung SBRT failed to show any difference in local control or median survival based on tumor T staging. ${ }^{11}$ The purpose of this study was to determine how tumor $\mathrm{T}$ stage affects the overall survival and local control rates in patients treated with lung SBRT for early-stage NSCLC.

\section{METHODS}

\section{Patient Eligibility and Characteristics}

Between March 2005 and January 2008, 60 patients with primary NSCLC were treated with SBRT at the University of Virginia after institutional review board approval for an institutional protocol. Forty patients 


$$
\begin{aligned}
& \text { Abbreviations and Acronyms } \\
& \begin{aligned}
\text { BED } & =\text { biologic equivalent dose } \\
\text { CI } & =\text { confidence interval } \\
\text { CT } & =\text { computed tomography } \\
\text { FDG } & =\text { fluorodeoxyglucose } \\
\text { GTV } & \text { gross tumor volume } \\
\text { HR } & =\text { hazard ratio } \\
\text { NSCLC } & =\text { non-small-cell lung cancer } \\
\text { PET } & =\text { positron emission tomography } \\
\text { PTV } & =\text { planning target volume } \\
\text { SBRT } & =\text { stereotactic body radiation therapy } \\
\text { SUV } & =\text { standardized uptake value } \\
\text { V20 } & =\text { volume of lung receiving } 20 \mathrm{~Gy}
\end{aligned}
\end{aligned}
$$

with peripheral primary NSCLC were identified and reviewed. Peripheral tumors were defined by the Radiation Therapy Oncology Group protocols as the primary tumor not touching a volume $2 \mathrm{~cm}$ in all directions around the proximal bronchial tree (distal $2 \mathrm{~cm}$ of the trachea, main stem bronchi, and lobar bronchi). The median patient age was 73 years (range, 54-87 years). All patients were required to undergo computed tomography (CT) of the chest and abdomen for clinical staging. No patients in our cohort underwent mediastinoscopy or endobronchial ultrasound owing to medical comorbidities. On the basis of CT imaging, patients were classified as having clinical stage I (T1-2 N0) according to the American Joint Committee on Cancer staging. Positron emission tomography (PET)-CT scans were obtained as part of the initial staging workup in $34(85 \%)$ patients. Histologic confirmation of cancer was obtained in all patients by either tissue biopsy or cytology. Patients with abnormal fluorodeoxyglucose (FDG) uptake (maximum standardized uptake value $[\mathrm{SUV}]>2.5$ ) in the mediastinum were not considered candidates for SBRT.

All patients were evaluated by a thoracic surgeon and radiation oncologist at our institution. Patients were treated with lung SBRT if they were considered to be in medically inoperable condition or if they refused surgery. Patients were considered to be medically inoperable owing to an underlying physiologic condition prohibiting a potentially curable resection based on a low probability of tolerating anesthesia, the operation, or the recovery period. Guidelines for inoperability were determined by the thoracic surgeon and typically included a predicted postoperative forced expiratory volume in 1 second of less than $30 \%$, severely reduced diffusion capacity greater $40 \%$ predicted, a performance status of 3 or greater, or severe cardiac disease. No patients received prior lung irradiation.

\section{Pretreatment Assessment and Follow-up}

Before SBRT was initiated, all patients underwent the following evaluation: physical examination, weight and performance status assessment, pulmonary function tests, and CT scan of the chest. After SBRT, followup was performed approximately 4 to 8 weeks after treatment and approximately every 3 months thereafter. CT of the chest was routinely obtained at 3-month intervals from the completion of radiotherapy. PET-CT was not routinely obtained before the initiation of therapy. Twelve patients underwent pretreatment and posttreatment PET-CT scans. The median time from the end of treatment until PET-CT acquisition was 9.8 months. Tumor response was defined as a decrease in the maximum tumor diameter by $30 \%$ on CT imaging or decrease in abnormal FDG uptake of the tumor by $20 \%$ as measured by maximum SUV. Local tumor recurrence was defined as a $20 \%$ increase in the largest tumor diameter on successive follow-up imaging at 3month intervals based on Response Evaluation Criteria in Solid Tumors (RECIST). ${ }^{12}$ Lobar recurrences were defined as local recurrences. Local re- currence as demonstrated by an increase in abnormal FDG uptake was required to correspond to an enlarging $\mathrm{CT}$ abnormality. Metastatic recurrence was defined as both regional nodal recurrence (N1 or N2) and distant systemic metastases. Distant recurrence was determined by imaging (CT or PET) and not confirmed pathologically unless amenable to percutaneous biopsy. Toxicity was graded using the Common Toxicity Criteria for Adverse Events (CTCAE v3.0).

\section{SBRT Planning}

All patients underwent treatment planning scans using free breathing helical CT imaging. Patients were immobilized using a "frameless"' semirigid evacuated bag system (Vac-Lok; MEDTEC, Orange City, Iowa). An isocenter was placed in the geometric center of the tumor. Isocenter coordinates were used for daily alignment for subsequent treatments. The gross tumor volume (GTV) was identified on each axial CT slice using pulmonary windowing. Solid components and adjacent ground-glass opacity were included in the volume. The clinical target volume was identical to the GTV. In patients who underwent pretreatment PET-CT scans, PET imaging was not used for treatment planning purposes. The planning target volume (PTV) was intended to account for setup variability and target motion. The volume was designed by expanding the GTV $0.5 \mathrm{~cm}$ in the radial direction and 1.0 $\mathrm{cm}$ in the cranial-caudal direction to account for tumor motion during a normal respiratory cycle as determined by the Radiation Therapy Oncology Group 0236 protocol. Normal tissue dose constraints were as follows: for the heart, trachea, and ipsilateral bronchus, a 30-Gy maximum point dose; for the esophagus, a 27-Gy maximum point dose; for the brachial plexus, a 24-Gy maximum point dose; and for the spinal cord, an 18-Gy maximum point dose.

Treatment planning was coordinated with both the thoracic surgeons and the radiation oncologists and performed using Hi-Art Helical TomoTherapy inverse planning software (Tomotherapy, Madison, Wis). Lung and bone density corrections were used for planning. The dose was prescribed such that $100 \%$ of the dose covered $95 \%$ of the PTV. The median prescribed dose in our cohort was $60 \mathrm{~Gy}$ (range, $42-60 \mathrm{~Gy}$ ) in 3 to 5 fractions. The median biologic equivalent dose (BED) for the cohort was $150 \mathrm{~Gy}$ (range, $78-180 \mathrm{~Gy}$ ). The selection of total doses and fractionation to the primary tumor was determined by constraints to the adjacent normal tissues. Figure 1 shows a typical SBRT treatment plan. The prescribed dose was not dictated by $\mathrm{T}$ stage or tumor size. Successful planning attempted to meet the following criteria: (1) conformality of the PTV as judged by the volume of the prescription isodose to the PTV should be less than 1.2 with minor deviations occurring 1.2 to 1.4 ; (2) any dose greater than $105 \%$ of the prescription should fall within the PTV: (3) heterogeneity of the PTV as judged by the dose to $5 \%$ of the PTV to the dose to $95 \%$ of the PTV should be less than 1.2; (4) volume of lung receiving 20 Gy should be less than $10 \%$ (V20).

\section{Statistical Analysis}

The follow-up was determined from the date of the final SBRT treatment to calculate median follow-up and Kaplan-Meier outcome data including local control and overall survival. SPSS (SPSS, Inc, Chicago, Ill) was used for statistical analysis. Log-rank tests were used to compare outcome data between T-stage groups. A Cox regression analysis was performed to adjust outcomes based on patient-specific data using multiple variables analyzed simultaneously.

\section{RESULTS}

The patient and tumor characteristics are listed in Table 1. The most common reason for inoperability was poor pulmonary reserve, which was identified in 26 patients (median forced expiratory volume in 1 second $33 \%$ of predicted, median diffusion capacity $30 \%$ of predicted). Other reasons 

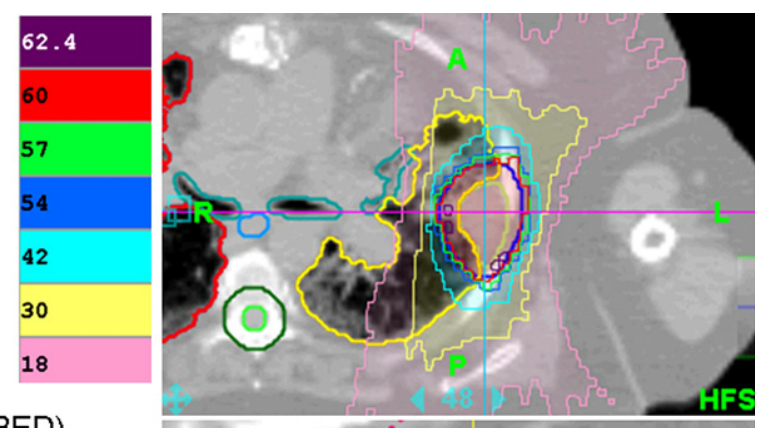

Biologic Equivalent Dose (BED)

\begin{tabular}{|c|c|c|}
\hline $\begin{array}{l}\text { Nominal } \\
\text { Dose (Gy) }\end{array}$ & $\begin{array}{l}\text { BED for 3 } \\
\text { Fractions }\end{array}$ & $\begin{array}{l}\text { BED for 5 } \\
\text { Fractions }\end{array}$ \\
\hline 60 & 180 & 132 \\
\hline 57 & 171 & 125 \\
\hline 54 & 162 & 118 \\
\hline 42 & 126 & 92 \\
\hline 30 & 90 & 66 \\
\hline
\end{tabular}

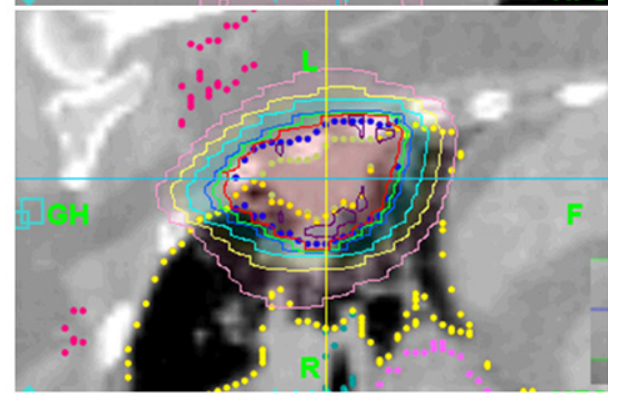

FIGURE 1. Representative helical TomoTherapy SBRT treatment plan with isodose lines and corresponding BED.

that patients were deemed to be in inoperable condition included severe coronary artery disease not amenable to revascularization (3 patients) or cardiac dysfunction with an ejection fraction less than 30\% (5 patients) and a Karnofsky performance status less than $70 \%$ (3 patients). Seventy percent of patients were dependent on supplemental oxygen. Three $(8 \%)$ patients refused surgical management of their disease including mediastinal staging with endobronchial ultrasound or mediastinoscopy. All patients completed intended radiotherapy without interruption. The median follow-up time for the entire cohort from the end of treatment was 12.5 months (range, 2-35 months). There was

TABLE 1. Patient and tumor characteristics

\begin{tabular}{lc}
\hline Median age $(\mathrm{y})$ & $73(54-87)$ \\
Median tumor diameter $(\mathrm{cm})$ & $2.3(0.9-6.5)$ \\
Tumor stage & \\
T1 & 27 \\
T2 & 13 \\
Median follow-up (mo) & $12.5(2.1-34.7)$ \\
T1 & $14.5(3-34.7)$ \\
T2 & $11.2(2.1-26)$ \\
& $P=.25$ \\
Median total dose (Gy) & $60(42.5-60)$ \\
T1 & $60(42.5-60)$ \\
T2 & $55(45-60)$ \\
& $P=.13$ \\
Median BED (Gy) & $150(78-180)$ \\
T1 & $150(78.6-180)$ \\
T2 & $150(85.5-180)$ \\
& $P=.07$ \\
\hline
\end{tabular}

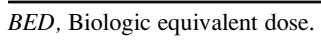

no statistical difference in follow-up, nominal radiation dose, or BED between the patients with $\mathrm{T} 1$ and $\mathrm{T} 2$ tumors (see Table 1).

The median tumor size was $23 \mathrm{~mm}$ (range, 9-50 mm) with $27(67 \%)$ and $13(33 \%)$ having T1 and T2 tumors, respectively. All tumors were peripheral in the lung. The median distance to the chest wall was $4 \mathrm{~mm}$ (range, 0-25 $\mathrm{mm}$ ). Twenty-six patients had primary tumors located in the upper lobe of the lungs, 12 in the lower lobe, and 2 in the right middle lobe.

The median PTV was $39.8 \mathrm{~mL}$ (range, 7.98-47.0 mL) for T1 tumors and $103 \mathrm{~mL}$ (range $40.5-196 \mathrm{~mL}$ ) for T2 tumors. Thirty-two $(80 \%)$ patients meet plan criteria for conformality with an index less than 1.2 . Eight $(20 \%)$ patients were considered to have minor deviations (conformality index 1.2-1.4). The mean conformality index was 1.17 (SD \pm 0.12). All plans met the criteria for heterogeneity with a mean heterogeneity index of 1.06 ( $\mathrm{SD} \pm 0.04)$. The mean V20 for the cohort was $8.08 \%(\mathrm{SD} \pm 3.4 \%)$. The median beam-on time per fraction was 22 minutes.

Toxicity data are listed in Table 2 . Twenty-three percent of patients $(9 / 40)$ had chest wall pain. The median time to onset of chest wall pain was 7.1 months (range, 0.6-32.3 months). The median time to resolution of chest wall pain was 4.6 months (range, $0.7-10.5$ months). Two (5\%) patients were identified as having a rib fracture as documented by chest CT. The median time to diagnosis of rib fracture was 20.6 months (range, 8.9-33.3 months). Symptomatic pulmonary complications (> grade 1) occurred in approximately $10 \%$ of patients, with 1 patient having grade 3 and grade 4 pneumonitis. No treatment-related deaths occurred. 
TABLE 2. Toxicity data

\begin{tabular}{lc}
\hline & No. $(\mathbf{n}=\mathbf{4 0})$ \\
\hline Chest wall pain & $9(23 \%)$ \\
Rib fracture & $2(5 \%)$ \\
Pneumonitis & 2 \\
$\quad$ Grade 1 & $5(12 \%)$ \\
Grade 2 & $1(2 \%)$ \\
Grade 3 & $1(2 \%)$ \\
& Median (range) \\
Onset of pain (mo) & $7.1(0.6-32.3)$ \\
Time to rib fracture (mo) & $20.6(8.9-33.3)$ \\
\hline
\end{tabular}

Of the 12 patients who underwent pretreatment and posttreatment PET-CT imaging, the median pretreatment maximum SUV was 6.0 (range, 1.0-16.2). Sixty-six percent $(8 / 12)$ had a decrease in their 9-month posttreatment maximum SUV with a median value of 2.0 (range, 0-10.9). The mean maximum SUV reduction was $56 \%$ (range, $18 \%-100 \%)$. Thirty-four percent of patients (4/12) had an increase in their tumor posttreatment maximum SUV with a mean increase of $38 \%$ (range, $24 \%-60 \%$ ).

Seven $(17 \%)$ patients had local tumor recurrence with or without distant recurrence. Eight patients (stage $\mathrm{T} 1=5$, stage $\mathrm{T} 2=3$ ) had distant recurrence, 2 of whom had documented local recurrence at the time of distant metastatic disease. Overall survival and local control (Figures 2 and 3) were determined for the entire cohort. The overall local control at 2 years for the entire cohort was $83 \%$. The mean recurrence-free survival for the entire cohort was 27.1 months (95\% confidence interval [CI], 22.6-31.6). Differences in local control were evaluated on the basis of tumor size and clinical $\mathrm{T}$ stage. Increasing size correlated with increased local failure (hazard ratio [HR], 1.904; 95\% CI, 1.130-3.210; $P=.016)$. Local control at 2 years for T1 tumors was $90 \%$, whereas local control was $70 \%$ for T2 tumors $(P=.035)$. The median recurrence-free survival for T1 and T2 tumors was 30.6 months $(95 \% \mathrm{CI}$, 26.9-34.2) and 20.5 months (95\% CI, 14.3-26.5), respectively $(P=.038)$. The mean time to local recurrence for $\mathrm{T} 1$ and T2 tumors was 16 and 7.5 months, respectively. Local control for both T-stage groups was independent of patient age (HR, 1.074; 95\% CI, 0.950-1.214), tumor histology (HR, 0.466; 95\% CI, 0.044-4.928), pretreatment or posttreatment PET SUV (HR, 0.996; 95\% CI, 0.7301.395), total radiation dose (HR, 1.143 ; 95\% CI, 0.734 1.779), or BED (HR, 1.578; 95\% CI, 0.78-3.863). The median survival for the entire cohort was 20.1 months $(95 \% \mathrm{CI}$, 18.7-28.4). The 1- and 2-year survivals were $85 \%$ and $45 \%$, respectively. Differences in overall survival were evaluated on the basis of tumor size and clinical $T$ stage. Increasing tumor size correlated with poorer overall survival (HR, 1.720; 95\% CI, 1.154-2.563; $P=.008$ ). The median survival for T1 and T2 tumors was 20 months $(95 \% \mathrm{CI}$, 20.1-31.6 months) and 16.7 months (95\% CI, 10.8-21.2 months), respectively $(P=.073)$. The 1 - and 2-year survivals for T1 tumors were $87 \%$ and $55 \%$, respectively.

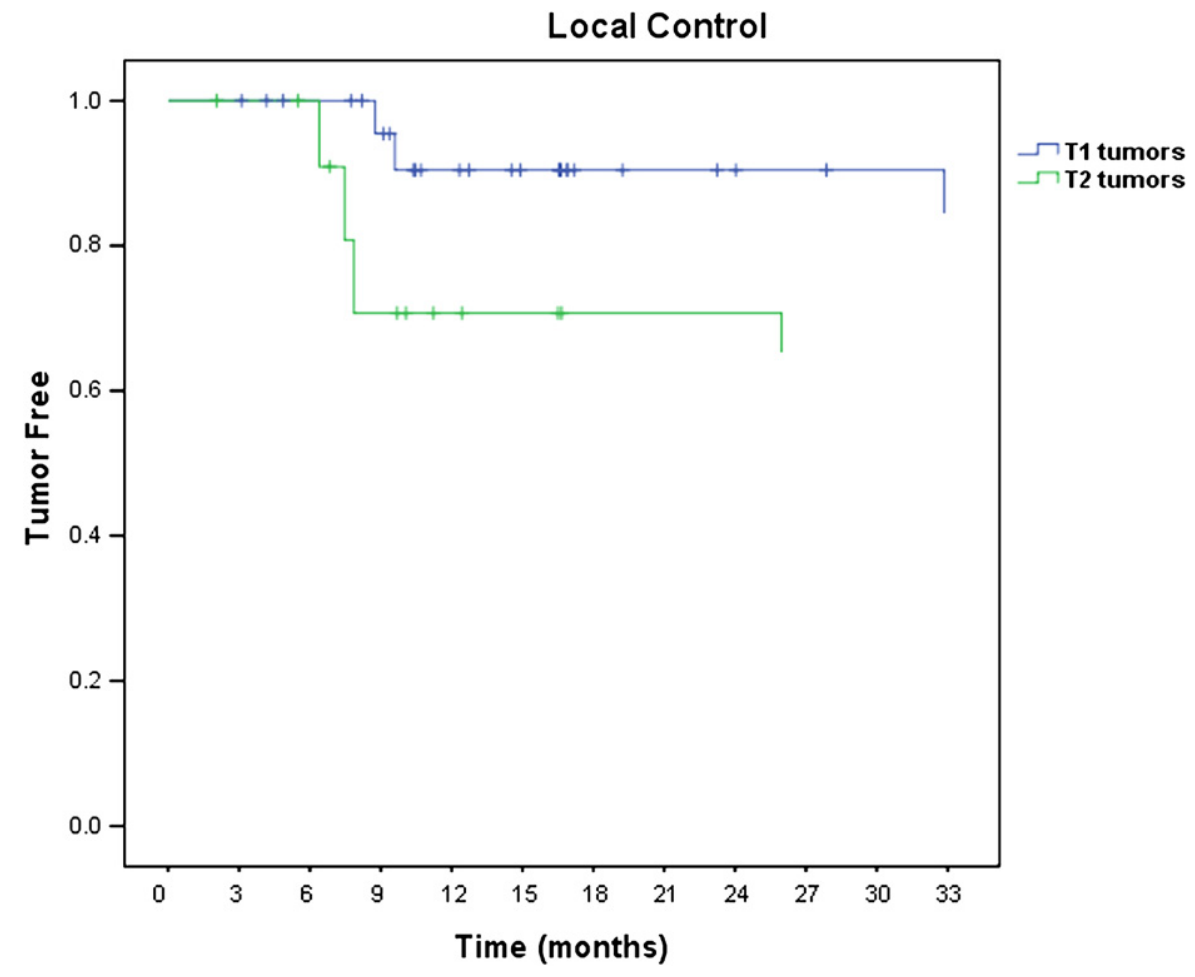

FIGURE 2. Local control by T-stage grouping. 


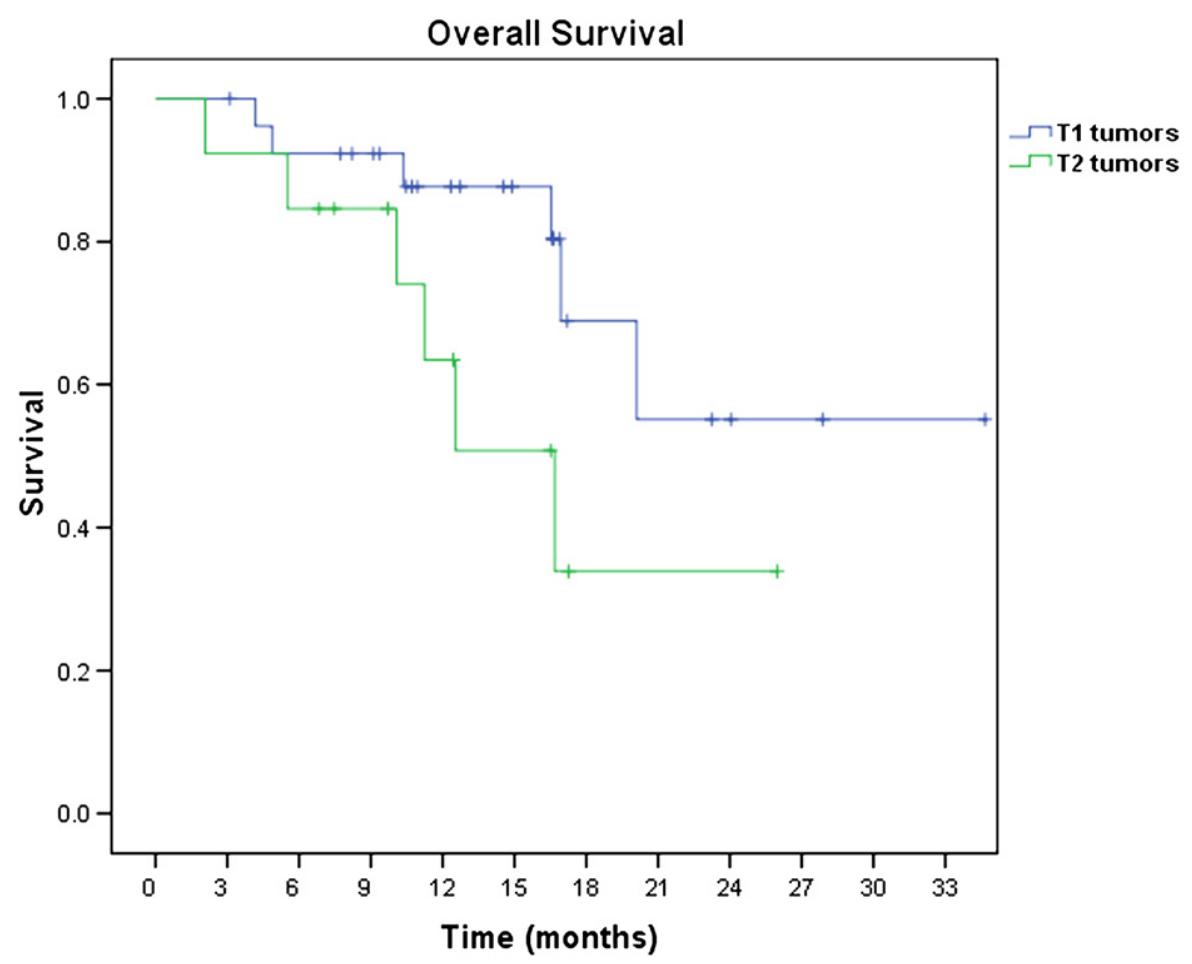

FIGURE 3. Overall survival by T-stage grouping.

The 1- and 2-year survivals for T2 tumors were $75 \%$ and $35 \%$, respectively.

\section{DISCUSSION}

Lung tumor size is an important factor for predicting outcomes with lung SBRT for medically inoperable patients. In our series, increasing tumor size correlated with local failure. Patients with T1 tumors had a $90 \%$ local control rate at 2 years whereas those with $\mathrm{T} 2$ disease had an inferior 2-year local control rate of $70 \%$. Small tumors (T1) also had a longer time to recurrence than did larger tumors (T2). Whether superior local control translates into a survival benefit is unclear. Our series demonstrates poorer overall survival when size was evaluated as a continuous variable. Smaller clinical $\mathrm{T}$ stage showed an estimated trend toward improved survival, although it did not quite reach statistical significance $(P<.07)$, likely secondary to the small sample size and short follow-up. Our median follow-up time was relatively short at 12.5 months with 3 patients whose follow-up period was less than 4 months, which limits the capture of local and distant failure. With longer follow-up, a more clear survival advantage may be demonstrated with T1 tumors as more occult metastases may present in patients with $\mathrm{T} 2$ tumors.

The treatment of primary NSCLC with SBRT has been advocated in medically inoperable patients. The local control with lung SBRT has been shown to be equivalent to limited surgical resection. The incidence of local recurrence after limited surgical resection with either wedge resection or segmentectomy has been reported to range from $10 \%$ to $27 \%$ in published series. ${ }^{2-5,13,14}$ Surgical data have shown that tumor $\mathrm{T}$ stage correlates with local failure, with most recurrences occurring within the first 2 years after resection. ${ }^{13,14}$ Published series using SBRT have reported similar local recurrence ranging from $10 \%$ to $30 \%$ with most recurrence occurring within the first year after treatment. ${ }^{8-11,15-17}$ Our series parallels other published data with an overall local recurrence rate of $17 \%$ at 2 years and a mean time to recurrence of approximately 12 months.

Tumor dose may play an important role in tumor sterilization and thus translate into improved local control and survival. Phase I and II studies have shown local control rates of $70 \%$ to $90 \%$ at 3 years and overall survivals of $50 \%$ to $60 \%$ using variable treatment regimens. ${ }^{8-11,15-17}$ Nominal total dose may underrepresent the biologic effectiveness of treatment; therefore, BED is often used as a surrogate to describe tumor dose. Our institutional cohort was treated with a median BED of 150 Gy (range, 78-180 Gy), which corresponds to doses reported in other published series. Initial studies from North America have advocated using a dose of 60 to 66 Gy delivered in 3 fractions (BED, 180 Gy). Yet other institutions from Europe and Asia have advocated using lower nominal doses of $45 \mathrm{~Gy}$ in 3 fractions (BED, $112.5 \mathrm{~Gy}$ ), 50 Gy in 5 fractions (BED, $100 \mathrm{~Gy}$ ), or $48 \mathrm{~Gy}$ in 4 fractions (105.6 Gy) with equivalent outcomes to larger BED schedules. ${ }^{13-17}$ Smaller fraction sizes may decrease rates of symptomatic 
pneumonitis, chest wall pain, rib fracture, or skin injury. Our data also show acceptable tumor control with minimal toxicity. Grade II and III pneumonitis was seen in only 2 patients with a mean V20 of $8 \%$. The incidence of chest wall pain was similar to that of other published reports, and only 2 patients had rib fractures. ${ }^{9,10}$

The implications of inferior local control and possibly survival with larger tumors may greatly affect the treatment approach with lung SBRT. A phase I series from Indiana University ${ }^{8}$ included tumors $7 \mathrm{~cm}$ or larger in greatest diameter. The original study design included dose escalation to determine the maximum tolerated dose, stratified by tumor size $(\leq 3 \mathrm{~cm}$ or $>3 \mathrm{~cm})$. The maximum tolerated dose for small tumors was 60 Gy in 3 fractions, whereas the maximum tolerated dose for tumors 5 to $7 \mathrm{~cm}$ was $66 \mathrm{~Gy}$ in 3 fractions. Local recurrence was observed most commonly at fraction sizes less than $1200 \mathrm{cGy}$. There was no observed difference in local control or toxicity between $\mathrm{T} 1$ and $\mathrm{T} 2 \mathrm{tu}-$ mors, although the authors state the study was not powered to detect a difference. A follow-up phase II study from the University of Indiana ${ }^{11}$ included $34 \mathrm{~T} 1$ tumors and $36 \mathrm{~T} 2$ tumors $7 \mathrm{~cm}$ or smaller. Small tumors were prescribed $60 \mathrm{~Gy}$ in 3 fractions and large tumors were prescribed $66 \mathrm{~Gy}$ in 3 fractions. A median follow-up of 50.2 months trended toward a worse survival for T2 tumors (24.5 months) compared with T1 tumors (38.7 months), although the difference was not significant statistically.

Chen and associates ${ }^{15}$ recently performed a retrospective evaluation of the efficacy of dose-individualized stereotactic body radiotherapy for patients with T1-3 NSCLC. A majority of patients were in stage T1. Sixty-five patients received 71.8 to 115.2 Gy of BED in 3.6- to 8.0-Gy daily fractions. No difference in local control or overall survival was seen between patients with $\mathrm{T} 1, \mathrm{~T} 2$, or $\mathrm{T} 3$ tumors.

Inoue and colleagues ${ }^{16}$ retrospectively reviewed clinical outcomes for clinically diagnosed small primary lung cancers in medically inoperable patients. Primary lung cancer was not confirmed pathologically. A total of 115 patients were treated with SBRT using a total dose of 30 to $70 \mathrm{~Gy}$ in 2 to 10 fractions. Ninety-three percent of the patients had T1 tumors. There was no observed difference in local tumor response on the basis of tumor size. The 3-year overall survival for tumors $20 \mathrm{~mm}$ or smaller was $89 \%$ versus $60 \%$ for patients with tumors larger than $20 \mathrm{~mm}$. This study suggests improved clinical outcome for smaller lesions although the authors point out this may partially be due to the inclusion of nonmalignant lesions in the cohort.

The impact of larger (T2) tumors with regard to treatment planning is currently unclear. Our data, as well as other series, suggest increased local failure compared with $\mathrm{T} 1$ tumors and possibly poorer overall survival. Dose and fractionation selection in our cohort was not influenced by tumor size but rather to limit treatment-related toxicity and meet dose constraints to the normal adjacent structures.
Although our data show no significant correlation with total dose or BED and local failure, dose escalation may be necessary above $60 \mathrm{~Gy}$. Initial work from Indiana University ${ }^{8,11}$ prescribed larger tumors $(>7 \mathrm{~cm}) 66 \mathrm{~Gy}$ in 3 fractions, corresponding to a BED of approximately $200 \mathrm{~Gy}$. The median fraction size in our cohort was $12 \mathrm{~Gy}$, corresponding to a BED of $150 \mathrm{~Gy}$. This dose differential may account for increased local failure for larger tumors in our patients. Another possibility is to address microscopic tumor extension. Prior studies have shown that CT imaging underestimates tumor extension based on pathologic correlates by as much as $12 \mathrm{~mm} .{ }^{18}$ The standard approach in lung SBRT is to make the clinical target volume equivalent to the GTV, therefore specifically not targeting microscopic disease. Although dose falloff is sharp with SBRT, the beam penumbra is thought to be sufficient to sterilize microscopic disease. This concept may need to be reconsidered with larger tumor margins for treatment.

The detection of metastatic disease before treatment is important in predicting patient survival. In our series, mediastinal lymph node involvement was ruled out with enhanced CT or PET-CT. The sensitivity and specificity of PET-CT for detecting mediastinal lymph nodes has been reported to be $84 \%{ }^{19}$ Studies have also suggested that mediastinal nodal involvement may be higher in larger tumors. ${ }^{13,14,19}$ Clinical experience with lung SBRT in the treatment of primary NSCLC has shown less than $5 \%$ of isolated N1 or N2 nodal failures in the PET-CT era, ${ }^{20}$ suggesting that surgical staging is not required in this subset of inoperable patients. Despite the potential utility of PET-CT for assessing mediastinal lymph nodes, the lack of mediastinal surgical staging may affect patient survival and distant failure by not detecting occult metastases. Thirty-four patients in our cohort underwent clinical staging with PET-CT and 6 patients did not, potentially affecting detection of metastatic disease at the time of treatment.

The utility of PET-CT scan with conventional radiotherapy has been widely established in predicting treatment outcome in NSCLC. ${ }^{21,22}$ Prospective studies have shown that posttreatment PET scan is highly predictive of survival compared with CT scans, tumor stage, or performance status. ${ }^{23}$ In the setting of high doses per fraction, the PET response after treatment is likely different compared with conventional radiotherapy. Hoopes and associates ${ }^{24}$ treated 58 patients with SBRT for early-stage lung cancer with SBRT. Twenty-eight patients had pretreatment and posttreatment PET-CT imaging. Fourteen percent of patients had percent abnormal activity in the primary tumor at 2 years without evidence of regional or distant failure. The University of Pittsburgh recently reported that in 28 patients treated with lung SBRT, persistently elevated FDG uptake was common despite tumor regression. ${ }^{25}$ With a median follow-up of 1 year, patients with stable disease had a mean decrease in SUV of $28 \%$, partial responders had 
a mean decrease of $48 \%$, and complete responders had a mean decrease of $94 \%$. Our data show mixed posttreatment SUV response after SBRT. Sixty-six percent of patients with pretreatment and posttreatment PET-CT had a mean decrease in SUV of $56 \%$, whereas $34 \%$ of our patients had a mean increase of $38 \%$. There was no correlation between local tumor control and posttreatment SUV, although our findings are limited by the small number of patient with both pretreatment and posttreatment PET-CT.

The results of our SBRT study in patients with medically inoperable early-stage NSCLC are clinically equivalent to prior studies ${ }^{7-11,15-17}$ regarding local control and overall survival. Our data suggest that increased tumor size predicts local failure after SBRT. Similar to other single institutional data, ${ }^{8,11,15,16}$ larger tumor size suggests a clinically significant decrement in overall survival, although not statistically significant. Although our study has inherent limitations to retrospective studies, including selection bias and a small sample size, the findings bring into question the utility of SBRT for larger tumors. SBRT may not be an adequate local therapy for $\mathrm{T} 2$ tumors and thus alternative treatment modalities should be explored. Radiofrequency ablation as a primary modality or in combination with SBRT may overcome underlying biologic resistance and sterilize microscopic disease. Similarly, combined modality therapies such as concurrent chemotherapy or targeted agents may be appropriate for larger tumors to improve local control and overall survival.

\section{CONCLUSIONS}

SBRT using helical, arc-based radiation therapy is a welltolerated and efficacious treatment for medically inoperable patients with early-stage peripheral NSCLC. Increasing tumor size correlates with poorer survival and local control. Patients with T2 tumors have higher rates of local recurrence than those with T1 tumors. Although not significant, T1 tumors trended toward improved overall survival. Tumor size is likely a significant factor in treatment response and may need to be taken into account during treatment planning. Options may include dose escalation, large treatment margins to cover microscopic disease, or combined modality therapies.

\section{References}

1. Mountain CF. A new international staging system for lung cancer. Chest. 1986; $89: 225 \mathrm{~S}-33 \mathrm{~S}$

2. Naruke T, Goya T, Tsuchiya R, Suemasu K. Prognosis and survival in resected lung carcinoma based on the new international staging system. J Thorac Cardiovasc Surg. 1988;96:440-7.

3. Dominguez-Ventura A, Cassivi SD, Allen MS, Wigle DA, Nichols FC, Pairolero PC, et al. Lung cancer in octogenarians: factors affecting long-term survival following resection. Eur J Cardiothoacr Surg. 2007;32:370-4.

4. Sanchez PG, Vendrame GS, Madke GR, Pilla ES, Camargo Jde J, Andrade CF, et al. Lobectomy for treating bronchial carcinoma: analysis of comorbidities and their impact on postoperative morbidity and mortality. J Bras Pneumol. 2006;32:495-504
5. Ginsberg RJ, Rubinstein LV. Randomized trial of lobectomy versus limited resection for T1 N0 non-small cell lung cancer. Ann Thorac Surg. 1995;60:615-23.

6. Coy P, Kennelly GM. The role of curative radiotherapy in the treatment of lung cancer. Cancer. 1980;45:698-702.

7. Dosoretz DE, Katin MJ, Blitzer PH, Rubenstein JH, Salenius S, Rashid M, et al. Radiation therapy in the management of medically inoperable carcinoma of the lung: results and implications for future treatment strategies. Int J Radiat Oncol Biol Phys. 1992;24:3-9.

8. Timmerman R, Papiez L, McGarry R, Likes L, DesRosier C, Frost S, et al. Extracranial stereotactic radioablation: results of a phase I study in medically inoperable stage I non-small cell lung cancer. Chest. 2003;124:1946-55.

9. Zimmermann FB, Geinitz H, Schill S, Grosu A, Schratzenstaller U, Molls M, et al. Stereotactic hypofractionated radiation therapy for stage I non-small cell lung cancer. Lung Cancer. 2005;48:107-14.

10. Fritz P, Kraus H, Blaschke T, Mühlnickel W, Strauch K, Engel-Riedel W, et al Stereotactic, high single-dose irradiation of stage I non-small cell lung cancer (NSCLC) using four-dimensional CT scans for treatment planning. Lung Cancer. 2008;60:193-9.

11. Fakiris AJ, McGarry RC, Yiannoutsos CT, Papiez L, Williams M, Henderson MA, Timmerman R. Stereotactic body radiation therapy for earlystage non-small-cell lung carcinoma: four-year results of a prospective phase II study. Int J Radiat Oncol Biol Phys. 2009;75:677-82.

12. Therasse P, Arbuck SG, Eisenhauer EA, Wanders J, Kaplan RS, Rubinstein L, et al. New guidelines to evaluate the response to treatment in solid tumors. J Natl Cancer Inst. 2000;92:205-16.

13. Konaka C, Ikeda N, Hiyoshi T. Peripheral non-small cell lung cancers $2.0 \mathrm{~cm}$ or less in diameter: proposed criteria for limited pulmonary resection based upon clinicopathological presentation. Lung Cancer. 1998;21:185-91.

14. Martini N, Bains M, Burt M, Zakowski M, McCormack P, Rusch V, et al. Incidence of local recurrence and second primary tumors in resected stage I lung cancer. J Thorac Cardiovasc Surg. 1995;109:120-9.

15. Chen Y, Guo W, Lu Y, Zoub B. Dose-individualized stereotactic body radiotherapy for T1-3N0 non-small cell lung cancer: long-term results and efficacy of adjuvant chemotherapy. Radiother Oncol. 2008;88:351-8.

16. Inoue T, Shimizu S, Onimaru R, Takeda A, Onishi H, Nagata Y. Clinical outcomes of stereotactic body radiotherapy for small lung lesions clinically diagnosed as primary lung cancer on radiologic examination. Int $J$ Radiat Oncol Biol Phys. 2009;75:683-7.

17. Baumann P, Nyman J, Hoyer M, Wennberg B, Gagliardi G, Lax I, et al. Outcome in a prospective phase II trial of medically inoperable stage I non-small-cell lung cancer patients treated with stereotactic body radiotherapy. J Clin Oncol. 2009;27: 3290-6.

18. Grills IS, Fitch DL, Goldstein NS, Yan D, Chmielewski GW, Welsh RJ, et al Clinicopathologic analysis of microscopic extension in lung adenocarcinoma: implications for defining the clinical target volume (CTV) for radiotherapy. Int J Radiat Oncol Biol Phys. 2005;63:S99-100.

19. Shim SS, Lee KS, Kim BT. Non-small cell lung cancer: prospective comparison of integrated FDG PET/CT and CT alone for preoperative staging. Radiology. 2005;236:1011-9.

20. Timmerman RD, Park C, Kavanagh BD. The North American experience with stereotactic body radiation therapy in non-small cell lung cancer. J Clin Oncol. 2007;2:S101-12.

21. Kubota K, Yamada S, Ishiwata K, Ito M, Ido T. Positron emission tomography for treatment evaluation and recurrence detection compared with $\mathrm{CT}$ in long-term follow-up cases of lung cancer. Clin Nucl Med. 1992;17:877-81.

22. Kubota K, Yamada S, Ishiwata K, Ito M, Fujiwara T, Fukuda H, et al. Evaluation of the treatment response of lung cancer with positron emission tomography and L-[methyl-11C]methionine: a preliminary study. Eur J Nucl Med. 1993;20: 495-501.

23. Mac Manus MP, Hicks RJ, Matthews JP, McKenzie A, Rischin D, Salminen EK, et al. Positron emission tomography is superior to computed tomography scanning for response-assessment after radical radiotherapy or chemoradiotherapy in patients with non-small-cell lung cancer. J Clin Oncol. 2003; 21:1285-92.

24. Hoopes DJ, Tann M, Fletcher JW, Forquer JA, Lin PF, Lo SS, et al. FDG-PET and stereotactic body radiotherapy (SBRT) for stage I non-small-cell lung cancer. Lung Cancer. 2007;56:229-34.

25. Coon D, Gokhale AS, Burton SA, Heron DE, Ozhasoglu C, Christie N. Fractionated stereotactic body radiation therapy in the treatment of primary, recurrent, and metastatic lung tumors: the role of positron emission tomography/computed tomography-based treatment planning. Clin Lung Cancer. 2008;9:217-21. 